\title{
Visualizing Betweenness Centrality of Process Area Net- works in Process Improvement Model for Service Provider Organization, CMMI-SVC
}

\author{
Shigeru Kusakabe *
}

\begin{abstract}
We expect process improvement models help effective facilitation for introducing advanced technology in providing superior services. In the literature of process improvement models, we have a claim that effective processes provide a vehicle for introducing and using new technology in a way that best meets the business objectives. However, process improvement models have their complexity and we need a clue to use them as our vehicle. In this paper, we analyze relationships among process areas in a process improvement model for service provider organizations, CMMI-SVC, by focusing on Related Process Areas components. We analyze process area networks whose nodes are process areas connected with their Related Process Areas components. Then, we visualize the result of the betweenness centrality analysis of the process area networks for each maturity level in order to analyze implication of the model.
\end{abstract}

Keywords: network analysis, process improvement model, service providing process

\section{Introduction}

Service providers need to properly introduce new technology for providing superior services to customers and end users. In the literature of process improvement models, we have a claim that effective processes provide a vehicle for introducing and using new technology in a way that best meets the business objectives. The article [1] claimed "The best part of assessment with respect to various standards is that a smart organization can use the assessment as a framework to evaluate how projects are done. And by conscious analysis rather than slavish adherence, the organization can plan and take steps that will improve its operation." We assume this claim should be also applicable to service providing projects and organizations. However, process improvement models have their complexity and we need a clue to use them as our vehicle.

We expect process improvement models help effective facilitation for introducing advanced technology into service providing processes. In this paper, we use an abstract model by which we can conduct process assessment and process improvement. Specifically, we

\footnotetext{
* Kyushu University, Fukuoka, Japan
} 
use an organization-level process model CMMI-SVC (Capability Maturity Model Integration for services), one of the CMMI ${ }^{\circledR}$ models (Capability Maturity Model ${ }^{\circledR}$ Integration)[2] as a reference model in this paper. CMMI models including CMMI-SVC are collections of best practices that help organizations to improve their processes, developed and maintained by the Software Engineering Institute (SEI) at Carnegie Mellon University (CMU).

Among the authorized service products for CMMI models managed by CMMI Institute, we use the official document for CMMI-SVC. The official document is publicly available and we can use it by ourselves for our internal use. Typically, it is necessary to clarify the weakness of the current process and the points where to apply new technology before actually introducing it to improve the current process. However, process improvement models have their complexity, and the document of CMMI-SVC is more than five hundreds pages in its volume, while we need to explain it to various stakeholders and convince them of its effectiveness. We need a clue to understand its effectiveness and to start to use it as our vehicle for introducing and exploiting new technology in a way that best meets the business objectives. Sometime it is useful to use intuitive information in making our decision. We expect having a bird's eye view is useful to find a clue to start to use the model as our vehicle. In order to gain a bird's eye view and facilitate intuitive understandings of the impact of introducing advanced technology in service providing processes, we analyze the betweenness centrality of the networks of process areas in CMMI-SVC.

The rest of this paper is organized as follows. In Section 2, we briefly introduce the CMMI-SVC model. Section 3 explains our approach to analyze the impact of introducing new technology through network analysis for the process area network using the Related Process Areas component in CMMI-SVC. We visualize the results of centrality analysis in process area networks for each maturity level and analyze its implications in Section 4. Section 5 introduces related work and Section 6 concludes this paper.

\section{Process Improvement Model CMMI-SVC}

In this section, we briefly introduce the CMMI-SVC model. The CMMI-SVC model is a collection of best practices from government and industry. The CMMI-SVC is one of the models generated from the CMMI Architecture and Framework. Currently, there exist three specific CMMI models.

- CMMI for Services (CMMI-SVC): Service establishment, management,

- CMMI for Development (CMMI-DEV): Product and service development, and

- CMMI for Acquisition (CMMI-ACQ): Product and service acquisition.

All CMMI models commonly contain 16 core process areas. These process areas cover basic concepts that are fundamental to process improvement in any area of interest (i.e., acquisition, development, services).

\subsection{CMMI-SVC}

The CMMI-SVC provides guidance for applying CMMI best practices in a service provider organization. In addition to the core process areas, CMMI-SVC has one shared process area, and seven service-specific process areas including one addition. The collection of best practices in the CMMI-SVC model focuses on activities for providing quality services to 
Table 1: Process areas in CMMI-SVC.

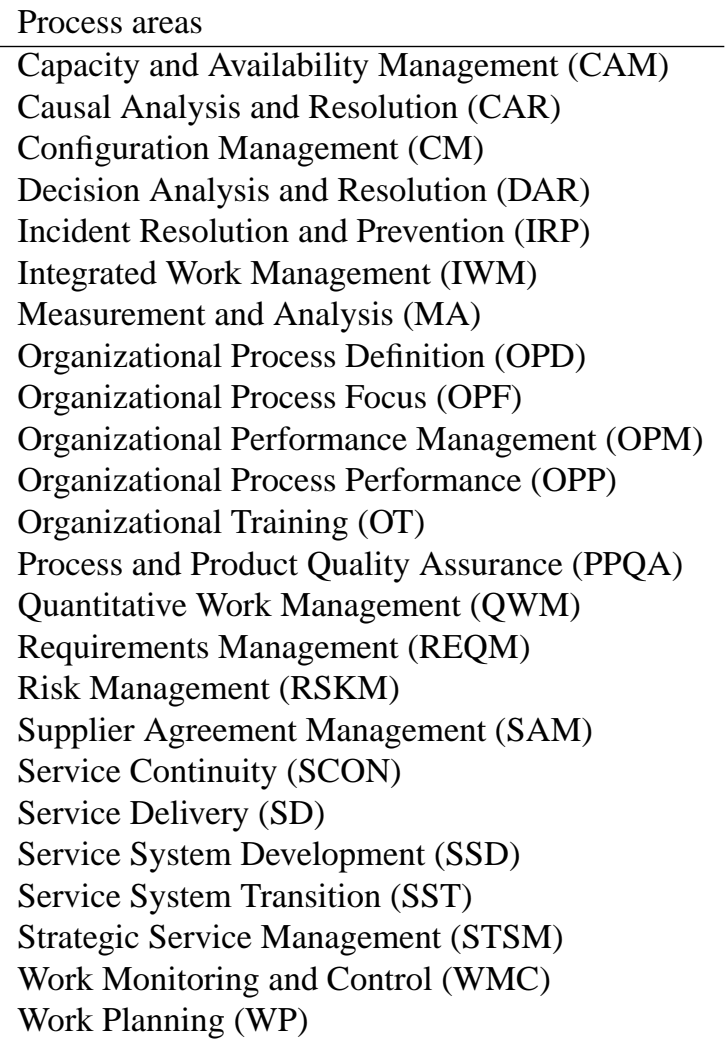

meet the needs of customers and end users. The CMMI-SVC has 24 process areas in total as listed in Table 1 in alphabetical order by acronym.

We have two different types of representation for these process areas in the CMMI framework. They provide two approaches to process improvement and these approaches are associated with two types of representation called continuous and staged representation, and corresponding the two different types of levels, capability levels and maturity levels. By using the continuous representation, we try to achieve the capability levels, and by using the staged representation we try to achieve the maturity levels. Table 2 shows the comparison of the capability levels and the maturity levels.

When using the staged representation, the organization incrementally improves a predefined set of process areas associated with the maturity levels. Table 3 shows the process

Table 2: Capability levels and maturity levels

\begin{tabular}{r|l|l} 
Level & Continuous representation & Staged representation \\
Capility levels & Maturity levels \\
\hline 0 & Incomplete & - \\
1 & Performed & Initial \\
2 & Managed & Managed \\
3 & Defined & Defined \\
4 & - & Quantitatively Managed \\
5 & - & Optimizing
\end{tabular}


Table 3: Maturity levels (ML) and the corresponding process areas in CMMI-SVC.

\begin{tabular}{r|l}
\multicolumn{1}{r|}{ ML } & Process areas \\
\hline \hline 2 & CM, MA, PPQA, REQM, SAM, SD, WMC, WP \\
\hline 3 & CAM, DAR, IRP, IWM, OPD, OPF, OT, RSKM, SCON, \\
& SSD, SST, STSM \\
\hline 4 & OPP, QWM \\
\hline 5 & CAR, OPM
\end{tabular}

Table 4: Categories in the continuous representation and the corresponding process areas in CMMI-SVC.

\begin{tabular}{l|l} 
Category & Process areas \\
\hline \hline Process Management & OPD, OPF, OPM, OPP, OT \\
\hline Project \& Work Management & CAM, IWM, QWM, REQM, \\
& RSKM, SAM, SCON, WMC, WP \\
\hline $\begin{array}{l}\text { Service Establishment } \\
\& \text { Delivery }\end{array}$ & IRP, SD, SSD, SST, STSM \\
\hline Support & CAR, CM, DAR, MA, PPQA
\end{tabular}

areas corresponding to each maturity level.

The CMMI framework is famous for this maturity level of the staged representation. In another representation, the continuous representation, process areas are organized into four categories: Process Management, Project \& Work Management, Service Establishment \& Delivery Support, and Support. Table 4 shows the categories in the continuous representation and the corresponding process areas.

When using the continuous representation, organizations select an individual process area or a set of process areas, and incrementally improve their processes corresponding to the selected process area or the set of process areas. When we recognize problems in our specific process areas and need to choose a method suitable to solve the problems, we are likely to adopt a process improvement approach of the continuous representation in using the improvement method.

\subsection{Components in Process Area}

Figure 1 shows model components and their relationships in the CMMI framework. Model components in the CMMI framework are grouped into three categories to indicate the way how to interpret the components: required, expected, and informative.

- Required Components: The specific and generic goals of a process area are the required components in the CMMI, which are essential to achieving process improvement in the process area.

- Expected Components: The specific and generic practices are the expected components in the CMMI. They describe the activities that are important in achieving required the CMMI components, the specific and generic goals. 
- Informative Components: These components play an important role in understanding the model and help model users understand the required and expected components. The model's informative material provides information necessary to achieve the correct understanding of goals and practices and thus cannot be ignored.

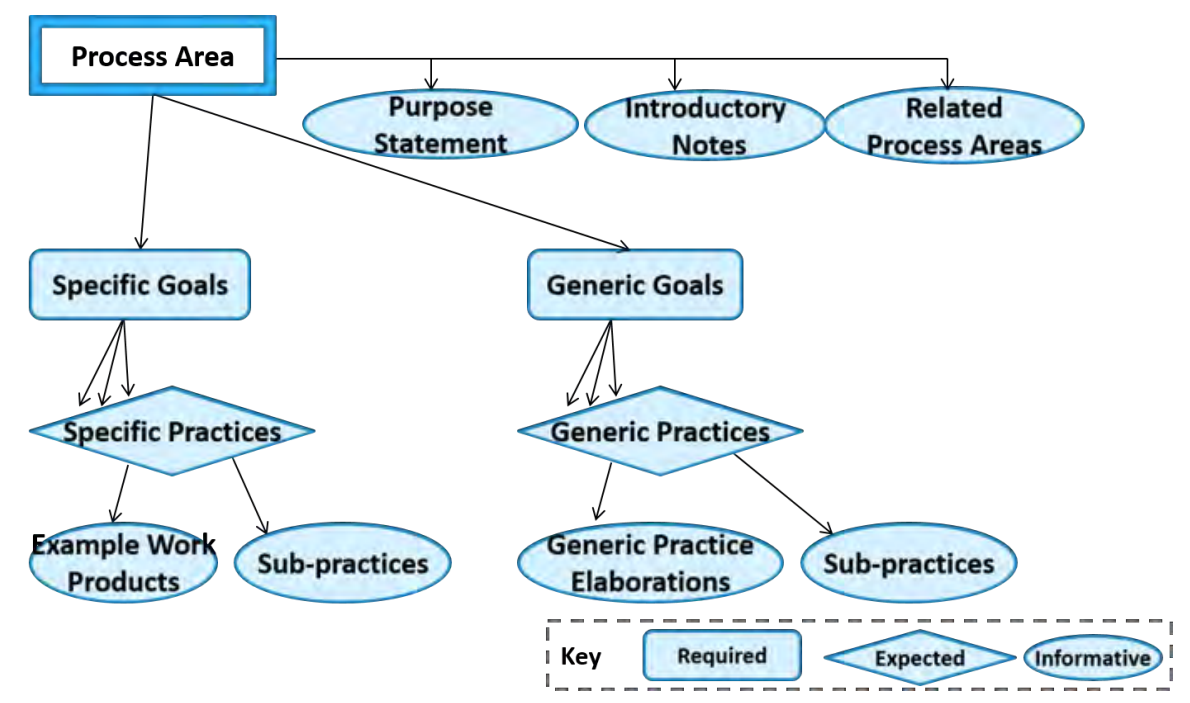

Figure 1: Model components and their relationships.

As we analyze the impact of new technology in process improvement from a bird's eye view through network analysis, we focus on the component, Related Process Areas, among Informative Components of CMMI-SVC in making a network representation, while abstracting away other components.

\section{Analysis of Process Area Network using Related Process Area Component}

In this section, we introduce a bird's eye view of CMMI-SVC through network analysis intending to facilitate sharing the perspective of introducing new technology among various stakeholders such as managers as well as engineers.

\subsection{Dependencies among Process Areas and Their Categories}

We expect that introducing new technology into a specific process area should have impact not only on the target process area but also other process areas including those in the categories other than the category to which the target process area belongs.

In order to extract implications, we analyze the dependencies among process areas by using a tool, Graphviz[3]. Figure 2 shows the dependencies between the process areas through the Related Process Areas component in CMMI-SVC within and across the bound-aries of four categories in the continuous representation. In the figure, the destination of an arrow is a Related Process Area component of the source of the arrow.

For example, as we can see from the figure, effects of improving process areas in Service Establishment \& Delivery category will propagate to process areas in other categories. 


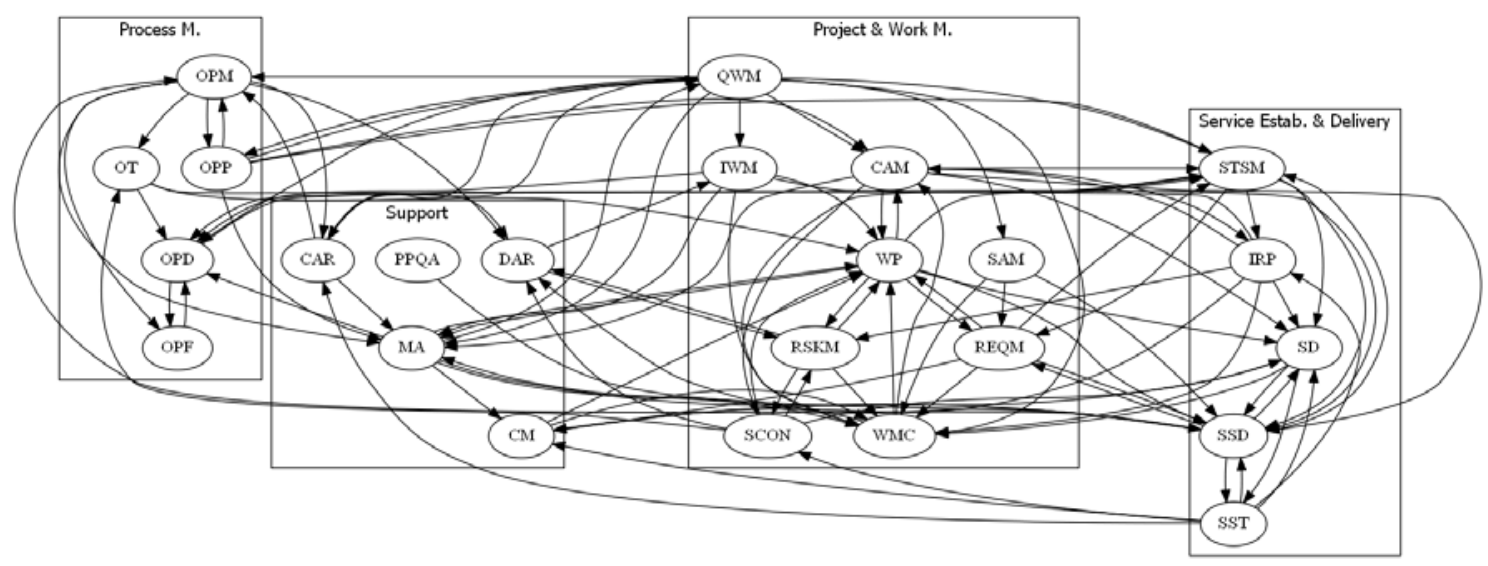

Figure 2: Process area dependencies based on the model component Related Process Areas within and among four categories of the continuous representation. The destination of an arrow is a Related Process Area component of the source of the arrow.

There exist dependencies among the process areas within the Service Establishment \& Delivery category and those outside of the category. This indicates process improvement for the process areas in the Service Establishment \& Delivery category will propagate to the process areas in other categories.

\subsection{Betweenness Centrality Value for Process Area}

In order to analyze chains of pair-wise relationships between process areas, we analyze relationships among process areas from the view point of the betweenness centrality, which corresponds to the number of times a node acts as a bridge along the shortest path between two other nodes. In Table 5, we show the ranking of process areas in terms of betweenness centrality value in ML2, ML3, ML4 and ML5. The table is sorted in the descending order of betweenness centrality value in ML5. We may select the top-ranked process area to begin our improvement effort. When we improve the top-ranked process area, the effect will widely propagate through the dependencies originating from it.

\section{Visualization of Betweenness Centrality for Maturity Level}

Our approach in this paper is intended to support a kind of hybrid approach in terms of the staged and continuous representations of the CMMI framework. We try to find a process area or a set of process areas in a maturity level of the staged representation, so that we can focus on the process area or the set of process areas as in continuous representation in the maturity level. We analyze sets of process areas in the staged representation in order to assist making a priority among process areas in the corresponding maturity level. In order to facilitate this kind of analysis we visualize the results of centrality analysis by using a tool, Gephi[4].

Figure 3 visualizes the analysis result of betweenness centrality in the process area network according to the Related Process Area component in each maturity level in CMMISVC. 
Table 5: Betweenness centrality values of process areas in terms of the Related Process Areas in each maturity level (normalized with the number of process areas in each maturity level). Process areas are sorted by ML5 values.

\begin{tabular}{l|r|r|r|r} 
Process Areas & ML2 & ML3 & ML4 & ML5 \\
\hline SSD & & 0.1832 & 0.1574 & 0.2126 \\
WP & 0.2381 & 0.1583 & 0.1582 & 0.1386 \\
MA & 0.0397 & 0.0440 & 0.1865 & 0.1308 \\
STSM & & 0.1533 & 0.1579 & 0.1293 \\
OPM & & & & 0.0970 \\
QWM & & & 0.1105 & 0.0956 \\
OPD & & 0.1062 & 0.0981 & 0.0677 \\
WMC & 0.1190 & 0.0809 & 0.1264 & 0.0638 \\
CAM & & 0.0641 & 0.0795 & 0.0568 \\
DAR & & 0.0615 & 0.0454 & 0.0392 \\
CAR & & & & 0.0370 \\
IRP & & 0.0290 & 0.0273 & 0.0361 \\
SD & 0.0159 & 0.0470 & 0.0440 & 0.0359 \\
SST & & 0.0410 & 0.0331 & 0.0341 \\
RSKM & & 0.0604 & 0.0475 & 0.0341 \\
IWM & & 0.0322 & 0.0383 & 0.0321 \\
REQM & 0.0635 & 0.0490 & 0.0465 & 0.0297 \\
SCON & & 0.0724 & 0.0657 & 0.0271 \\
OPP & & & 0.0000 & 0.0121 \\
OT & & 0.0099 & 0.0081 & 0.0077 \\
CM & 0.0238 & 0.0065 & 0.0077 & 0.0037 \\
SAM & 0.0000 & 0.0000 & 0.0027 & 0.0024 \\
OPF & & 0.0000 & 0.0000 & 0.0007 \\
PPQA & 0.0000 & 0.0000 & 0.0000 & 0.0000
\end{tabular}


- ML2: Managed

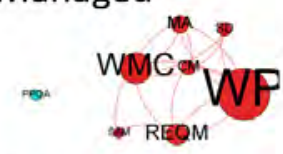

- ML3: Defined

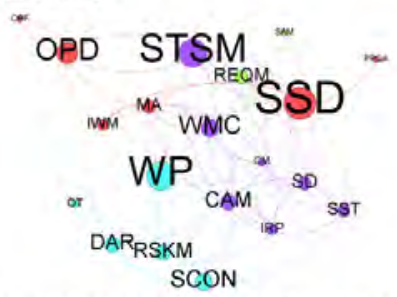

- ML4: Quantitatively Managed

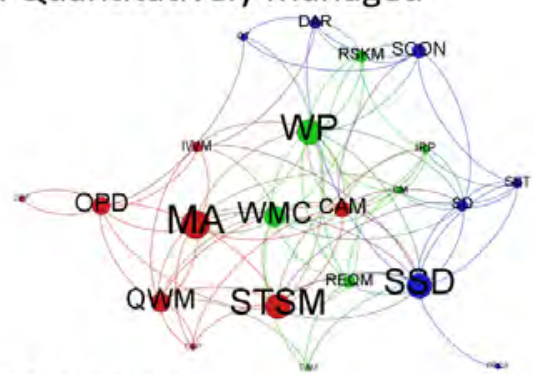

- ML5: Optimized

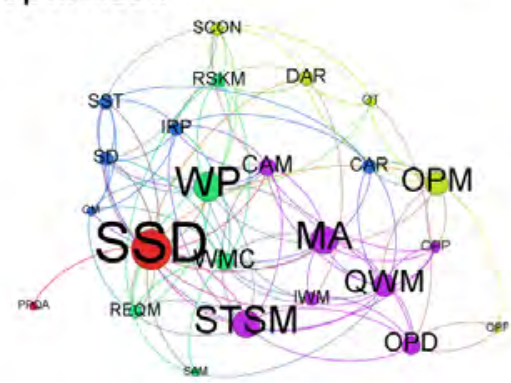

Figure 3: Visualization of betweenness centrality in the process area networks according to the Related Process Area component by maturity levels in CMMI-SVC. 
As we can see from these figures, Service System Development (SSD) seems to have a central role in ML3 and higher from a betweenness centrality view. Since SSD is a process area on development, this may indicate we need to achieve a high maturity level and/or a high capability level in CMMI-DEV as well as CMMI-SVC. This may lead to improvement using hierarchically related process templates such as a team level software development process, which assume a disciplined personal level software development process[5] [6] [7] [8].

We can also generate other hypotheses from the figure.

- In ML2, Work Planning (WP) seems more important than other process areas such as Service Delivery (SD).

- In ML3, Strategic Service Management (STSM) becomes important, as well as planning.

- In ML4, Measure and Analysis (MA) becomes important, as well as newly added Quantitative Work Management (QWM).

- In ML5, Organizational Performance Management (OPM) becomes important.

As far as the author's knowledge goes, there exist no case reports directly supporting our hypotheses on CMMI-SVC based on network analysis. However, there exist reports on critical success factors in improving service processes analyzed by using other approaches, like [9]. We will try collecting evidence and verifying our hypotheses by using such results that may not directly support our hypotheses.

\section{Related work}

There exist proposals regarding to the analysis of the model components in CMMI-DEV for development organizations [10] [11] [12], while we analyzed CMMI-SVC for service providing organizations.

In [11], the authors focused on the specific practices in the model. The CMMI-DEV official document does not provide any explicit recommendations about which specific practices can or should be implemented before other specific practices. They identified dependencies between specific practices within each process area, and between the process areas only for ML 2. They analyzed the text of the CMMI specification to identify every work product produced and used by every specific practice in ML 2.

Although we can analyze similar aspects for CMMI-SVC, we used a bird's eye view in order to share the perspective in introducing new technology among various stakeholders including managers as well as engineers. The network seems busy even for a single process area in their work. This kind of detailed information may not be useful in a top down approach, and may be difficult in scaling for large process models. However, dependency analysis of this level seems effective when we analyze the impact of new technology on detailed practices and work products within a limited range. Thus, we will try a hybrid and hierarchical approach in our future work.

\section{Concluding Remarks}

In this paper, we analyzed the process area networks using Related Process Areas component in the process improvement model CMMI-SVC in order to provide a perspective of the 
impact in introducing advanced technology in service providing processes. We expect using the standard process model as a reference can facilitate sharing of common understandings for the advantages of introducing advanced technology. We used network analysis to find key process areas which may play an important role in our intended improvement. We examined betweenness centrality in the networks of process areas for each maturity level, and discussed the implications embedded in the process improvement model which is a collection of best practices from government and industry.

\section{Acknowledgment}

This work was partly supported by KAKENHI, Grant-in-Aid for Scientific Research(S) 24220001 .

\section{References}

[1] M. E. Fayad and M. Laitinen. Process Assessment Considered Wasteful, CACM, Vol. 40, No. 11, 1997, pp.125-128.

[2] CMMI, http://cmmiinstitute.com/

[3] Graphviz - Graph Visualization Software, http://www.graphviz.org

[4] Gephi, an open-source software for visualizing and analyzing large networks graphs, https://gephi.org

[5] J. McHale, and D. Wall. Mapping TSP to CMMI, Carnegie Mellon University Software Engineering Institute Technical Report CMU/SEI-2004-TR-014, 2005.

[6] Team Software Process, http://www.sei.cmu.edu/tsp/

[7] W. S. Humphrey. Introduction to the Team Software Process, Addison-Wesley, 2000.

[8] W. S.Humphrey. PSP: A Self-improvement Process For Software Engineers, AddisonWesley, 2005.

[9] T. Diirr and G. Santos. Improvement of IT service processes: a study of critical success factors, Journal of Software Engineering Research and Development 2:4, 2014, online.

[10] P. Monteiro, R. Machado, R. Kazman and C. Henriques. Dependency analysis between CMMI process areas, Proc. of International Conference on Product-Focused Software Process Improvement (PROFES'10), 2010, pp.263-275.

[11] X. Chen, M. Staples and P. Bannerman. Analysis of Dependencies between Specific Practices in CMMI Maturity Level 2, In Proc. of EuroSPI Conference, 2008, pp. 94105.

[12] S. Kusakabe, H. Lin, Y. Omori, and K. Araki, Generating Supportive Hypotheses in Introducing Formal Methods using a Software Processes Improvement Model, Proc. of the ICSE 2014 Workshops - 2nd FME Workshop on Formal Methods in Software Engineering (FormaliSE 2014), 2014, pp.24-30. 